\title{
Large Area Full-Field Optical Coherence Tomography and its Applications
}

\author{
Shoude Chang ${ }^{*}$, Sherif Sherif, Youxin Mao and Costel Flueraru
}

Optics Group, Institute for Microstructural Sciences, National Research Council Canada, 1200 Montreal Road, Ottawa, K1A 0R6, Canada

\begin{abstract}
Optical Coherence Tomography (OCT) can perform high resolution, cross-sectional tomographic imaging of the internal structure of materials and biological samples. Most of the OCT systems use fibre probe based technology, in which a 3-axis scanning mechanism is needed. As the fibre output aperture is of the order of a micron, the working area of OCT system is normally about hundreds of squared microns. Working directly on a 2D region, Full-Field OCT (FF-OCT) system has parallel processing ability. Because the size of the image does not pose a serious challenge, FF-OCT can be easily used to image a large working area. This paper discusses system design and implementation for a large area FFOCT. Different applications, 3D intensity imaging, multiple-layer information extraction and fake-fingerprint detection, are described. To further reduce the cost of system and increase and the resolution of depth, FF-OCT system using white light is also introduced.
\end{abstract}

Keywords: Full-field optical tomography, optical image processing, 3D imaging, white light OCT system.

\section{INTRODUCTION}

Optical coherent tomography (OCT), developed in early 90 's [1], is a technique that permits high-resolution crosssectional imaging of an object. During the past decade, the OCT technology has attracted attention of many researchers. New OCT imaging theories and applications are being developed and reported on an increasing scale [2-4].

OCT systems can be roughly grouped into two types: Fibre Based OCT (FB-OCT) and Full-Field OCT (FF-OCT). For each of them, it can be further divided by two kinds: Time-Domain OCT (TD-OCT) and Fourier Domain or Swept-Source OCT (FD-OCT, SS-OCT). TD-OCT has the advantage of large dynamic range of the signal [5] and almost unlimited scanning depth. FD-OCT [6, 7] and SS OCT $[8,9]$ have better signal noise ratio and require no depth scanning. Most of the existing OCT systems use FB-OCT technology, in which point-scanning mechanism is performed. Depth scanning is achieved by the longitudinal translation of a reference mirror, and a sectional image is obtained by using scanning mirrors that laterally scan a focused probe beam on a test sample. Such 3-axis scanning makes the system slow and cumbersome. FD-OCT and SSOCT do not need depth scan, however, a grating and an array sensor (FD-OCT), or a scan in wavelength (SS-OCT) is required. To increase the acquisition speed and eliminate the need for 2D lateral scanning, parallel detection schemes have been investigated. A parallel OCT system illuminates an entire 2D target and collects light from all pixels simultaneously. These parallel OCT systems are usually called fullfield OCT (FF-OCT) systems. A few OCT systems working directly on 2D full-field images were reported [10-13]. As the fibre output aperture is about a micron leveling size, the

*Address correspondence to this author at the Optics Group, Institute for Microstructural Sciences, National Research Council Canada, 1200 Montreal Road, Ottawa, K1A 0R6, Canada;

E-mail: Shoude.Chang@nrc-cnrc.gc.ca working area of FB-OCT system is normally about hundreds of squared microns. Because FF-OCT has parallel processing ability, the size of the image does not really pose a serious challenge. Thus FF-OCT can be used to image a relatively large area.

This paper discusses system design and implementation as well as algorithms used for large area FF-OCT. Applications such as 3D intensity imaging, multiple-layer extraction and fake-fingerprint detection, are described. To further reduce the cost of the system, and increase and the resolution of depth, the design and fabrication of a FF-OCT system using white light is also introduced.

\section{THEORETICAL ASPECTS OF FULL-FIELD OCT}

For low-coherence light sources, the electric fields of sample and reference beams can be expressed by $E_{S}(t)$ and $E_{R}(t-\tau)$ :

$E_{S}(t)=1 / 2 \int_{-\infty}^{\infty} S_{S}(\omega) \exp (i \omega t) \mathrm{d} \omega$,

$E_{R}(t-\tau)=1 / 2 \int_{-\infty}^{\infty} S_{R}(\omega) \exp [i \omega(t-\tau)] \mathrm{d} \omega$,

where, $S_{S}(\omega)$ and $S_{R}(\omega)$ are the spectral functions of $E_{S}(t)$ and $E_{R}(t)$, respectively. The intensity of the interference signal is

$\mathrm{I}_{\mathrm{d}}(\tau)=\mathrm{E}_{\mathrm{S}}{ }^{2}+\mathrm{E}_{\mathrm{R}}{ }^{2}+1 / \pi \operatorname{Re} \int_{-\infty}^{\infty} S_{S}(\omega) S_{R}(\omega) \exp (\mathrm{i} \omega t) \mathrm{d} \omega$. (3)

Considering both the $S_{S}(\omega)$ and $S_{R}(\omega)$ to have a Gaussian spectral distribution defined by

$S(\omega)=S_{0} \exp \left(-2 \ln 2\left(\omega-\omega_{0}\right)^{2} / \Delta \omega^{2}\right)$,

the intensity of the interference signal is then

$I_{d}(\tau)=E_{S}{ }^{2}+E_{R}{ }^{2}+\left[S_{S 0} S_{R 0} \Delta \omega / \sqrt{ }(4 \pi \ln 2)\right] \exp \left[-\Delta \omega^{2} \tau^{2} /\right.$

$(16 \ln 2)] \cos \left(\omega_{0} \tau\right)$ 
$=I_{S}+I_{R}+I_{T} \exp \left[-4 \ln 2 \tau^{2} /\left(\Delta \tau^{2}\right)\right] \cos \left(\omega_{0} \tau\right)$,

where $I_{T}=S_{S 0} S_{R 0} \Delta \omega / \sqrt{ }(4 \pi \ln 2)$, and $\Delta \tau=8 \ln 2 / \Delta \omega$.

The time delay $\tau$ is determined by the difference of in optical path between sampling surface $l_{S}$ and reference surface $l_{R}$ :

$\tau=2\left(1_{\mathrm{R}}-1_{\mathrm{S}}\right) / \mathrm{c}$.

As the sample contains multiple flat layers, the light reflections from other layers also introduce interference. In this case,

$I_{d}(\tau)=\Sigma I_{S}+\sum I_{R}+\sum I_{T i} \exp \left[-4 \ln 2\left(\tau-\tau_{\mathrm{i}}\right)^{2} /\left(\Delta \tau^{2}\right)\right] \cos \left[\omega_{0}\left(\tau-\tau_{\mathrm{i}}\right)\right]$,

where $\tau_{\mathrm{i}}$ is the time delay of the $i^{\text {th }}$ layer. To extract the image of interest layer from others, the distance between each layer, $L_{L}$, must be larger than the resolution of the OCT system, i.e.,

$L_{L}>0.44 \lambda^{2} / \Delta \lambda$,

where $\lambda$ is the central wavelength and $\Delta \lambda$ is the FWHM of the light source. The time delay $\tau$ in Eq. (7) is usually generated by changing the reference arm length. All the tomographic images at different layers can be extracted one by one by changing this time delay.

For the full-field interference, at a position where $\tau=\tau_{\mathrm{i}}$, the Eq.(7) can be expressed by a 2D function

$I_{d}(x, y)=I_{0}(x, y)+A_{i}(x, y) \cos [\phi(x, y)]$,

where $I_{0}(x, y)$ is the background image, which is the intensity summation of all layer images; $\cos ($.$) represents the interfer-$ ence pattern, a function of variables $x$ and $y . A_{i}(x, y)$ is the tomographic image of $i^{\text {th }}$ layer.

To extract the tomogram $A_{i}(x, y)$, one needs to remove the background image $I_{0}(x, y)$, and eliminate the interference factor $\cos ($.$) . In full-field OCT, these two procedures become$ more difficult than in the point-scan based OCT system. Firstly, the signal-to-noise ratio gets worse due to the energy of the light energy spreading to a $2 \mathrm{D}$ area instead of focusing on a point. Secondly, the lateral interference fringes have to be removed, which is not needed in point-scan based OCT system.

\section{ALGORITHMS USED IN FULL FIELD OCT}

To retrieve the information hidden in the interference images, the interference fringes, represented by $\cos [\phi(x, y)]$, have to be removed through some processing. In [14], a method of removing fringes by using two $\pi / 2$ phase-shifted images $I_{1}(x, y), I_{2}(x, y)$ and a background image $I_{0}(x, y)$ is described. The tomogram can be resolved by

$A_{i}(x, y)=\left\{S_{I}(x, y)^{2}(x, y)+S_{2}(x, y)^{2}\right\}^{1 / 2}$.

where, $S_{1}(x, y)=I_{1}(x, y)-I_{0}(x, y), S_{2}(x, y)=I_{2}(x, y)-I_{0}(x, y)$.

The background image $I_{0}(x, y)$ can be obtained before or after the scanning. Two $\pi / 2$ phase-shifted images $I_{l}(x, y)$, $I_{2}(x, y)$ can be acquired either by using two cameras, as illustrated in Fig. (1d), or by grabbing one after another with a phase-shift in depth. However, when the phase-shift is an arbitrary value but not $\pi / 2$, the following formula should be used [13]

(a)

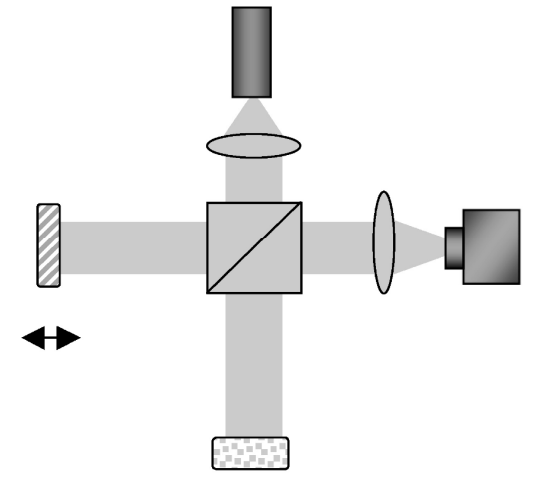

(b)

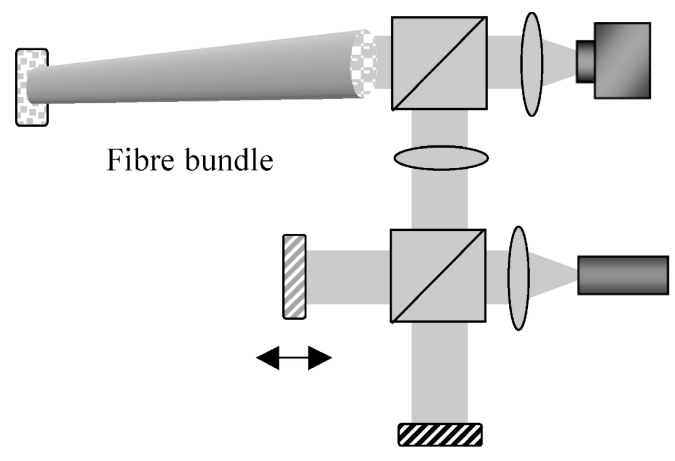

(c)

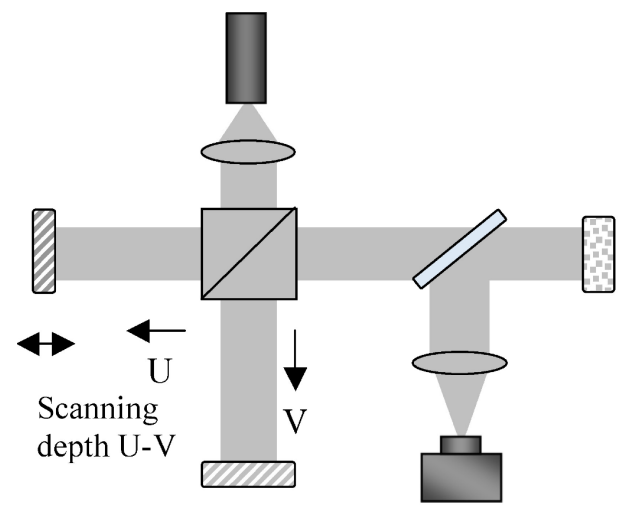

(d)

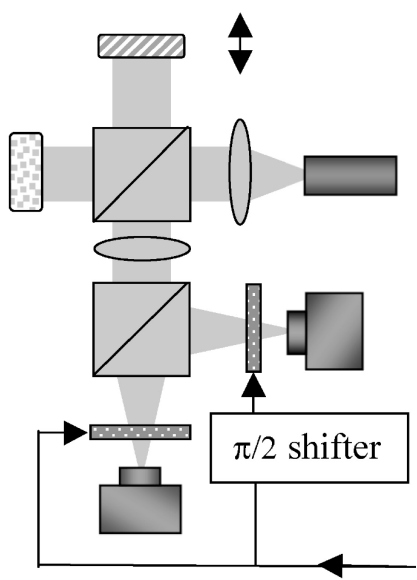

Fig. (1). Schematic diagrams of four FF-OCT systems. 
$A_{i}(x, y)=\left\{S_{I}^{2}(x, y)+\left[\left(S_{2}(x, y)-S_{I}(x, y) \cos \varphi\right) / \sin \varphi\right]^{2}\right\}^{1 / 2}$. (14)

However, both Eq.(13) and (14) need to know the phaseshift angle a priori. To solve the $A_{i}(x, y)$ without knowing the value of phase-shift, more phase-shift images have to be presented. Two algorithms can be derived from the 4-step or 5step phase-shift equations, which were originally proposed to find the interference phase function $\phi(x, y)$ in $70 \mathrm{~s}$. The tomographic image can be solved by Carré equations $[14,15]$ of 4-step phase-shift images:

$$
\begin{gathered}
A_{i}(x, y)=\left[\left(\frac{I_{l}(x, y)-I_{4}(x, y)+I_{2}(x, y)-I_{3}(x, y)}{8 \sin \varphi \cos ^{2} \varphi}\right)^{2}+\right. \\
\left.\left(\frac{I_{l}(x, y)+I_{4}(x, y)-I_{2}(x, y)-I_{4}(x, y)}{\cos \varphi \sin ^{2} \varphi}\right)^{2}\right]^{1 / 2} \\
\operatorname{tg} \varphi=\left\{\frac{3\left[I_{2}(x, y)-I_{3}(x, y]\right)-\left[I_{1}(x, y)-I_{4}(x, y)\right]}{I_{1}(x, y)-I_{4}(x, y)+I_{2}(x, y)-I_{3}(x, y)}\right\}^{1 / 2}
\end{gathered}
$$

wherein $I_{I}(x, y) \sim I_{4}(x, y)$ are four consequential images with a constant phase step $\varphi$. When $\varphi=n \pi / 2$, where $n$ is an integer, a correct table has to be used to avoid the presence of singularity.

As for the 5-step method, Hariharan equations [16] could be used to obtain the tomogram, which is expressed as

$$
\begin{aligned}
& A_{i}(x, y)=\left[\left(\frac{I_{2}(x, y)-I_{4}(x, y)}{2 \sin \varphi}\right)^{2}+\right. \\
& \left.\left(\frac{2 I_{3}(x, y)-I_{5}(x, y)-I_{l}(x, y)}{4 \sin ^{2} \varphi}\right)^{2}\right]^{1 / 2}
\end{aligned}
$$

where, $\varphi$ can not be $n \pi$.

$$
\tan \varphi=\frac{2\left[I_{2}(x, y)-I_{4}(x, y)\right]}{3 I_{3}(x, y)-I_{5}(x, y)-I_{I}(x, y)}
$$

Dubois et al. proposed a 4-integration buckets algorithm [17] [18], in which 4 images with circular-shifted phase angles $0, \pi / 2, \pi, 3 \pi / 2$ are acquired. Analyzed by using Bessel functions of first kind, $J_{n}$, the tomogram is obtained by

$A_{i}(x, y)=k\left\{\left[I_{I}(x, y)+I_{4}(x, y)-I_{2}(x, y)-I_{3}(x, y)\right]^{2}+\left[I_{I}(x, y)-I_{4}(x, y)\right.\right.$
$\left.\left.-I_{2}(x, y)+I_{3}(x, y)\right]^{2}\right\}^{1 / 2}$,

where $k$ is a coefficient, a function of $J_{n}$. Considering the phase-shift angle between each step is $2 \varphi$ in equations (16), when $2 \varphi=n \pi / 2$, i.e., $\varphi=n \pi / 4$, it is easy to show that Eq. (11) is equivalent to Eq. (15). Either 4-step or 5-step algorithm has to calculate the angle of phase step for each pixel in the captured interference images. The computation is relatively heavy, particularly when the image is large, since algorithms are pixel-based operations.
Chang et al.. proposed a derivative-based algorithm for FF-OCT [19]. In fact, Eq. (9) of an OCT interferometer is a function of time variable when the interferometer scans axially through a sample,

$I(x, y, t)=I_{0}(x, y)+A_{i}(x, y, t) \sin [\omega t+\phi(x, y)]$,

where $\omega=2 \pi f, f$ represents the central frequency of the light source. When the light source is a low-coherence source, as the case in most of the OCT devices, Eq. (22) becomes

$I(x, y, t)=I_{0}(x, y)+\mathrm{C}_{\mathrm{r}}(x, y, t) A_{\mathrm{i}}(x, y, t) \sin [\omega t+\phi(x, y)]$,

where $\mathrm{C}_{\mathrm{r}}(x, y, t)$ is the envelope of the autocorrelation of the light source. $\mathrm{C}_{\mathrm{r}}(x, y, t)$ is usually a Gaussian distribution, and its coherence length determines the axis resolution of an OCT system. However, when a small shift $\mathrm{d}$ is made, $\mathrm{d}<<$ Dc, $\mathrm{C}(x, y, t)$ can be considered as 1 , and the tomography of the current layer $A_{\mathrm{i}}(x, y, t)$ can be considered constant, i.e., $A_{\mathrm{i}}(x, y, t)=A_{\mathrm{i}}(x, y)$. In this assumption, the first, second and third derivatives of $I(x, y, t)$ with respect to scanning $t$ can be expressed by

$I_{t}{ }^{\prime}(x, y, t)=\omega A_{i}(x, y) \cos [\omega t+\phi(x, y)]$,

$I_{t}{ }^{\prime}{ }^{\prime}(x, y, t)=-\omega^{2} A_{i}(x, y) \sin [\omega t+\phi(x, y)]$,

$I_{t}{ }^{\prime, ~}{ }^{\prime}(x, y, t)=-\omega^{3} A_{i}(x, y) \cos [\omega t+\phi(x, y)]$,

respectively. Combining them by

$I_{t}{ }^{,{ }^{2}}{ }^{2}-I_{t}{ }^{, ",}, \times I_{t}{ }^{\prime}=\omega^{4} A_{i}{ }^{2}(x, y) \sin ^{2}[\omega t+\phi(x, y)]+\omega^{4} A_{i}{ }^{2}(x, y)$

$\cos ^{2}[\omega t+\phi(x, y)]$

$=\omega^{4} A_{i}^{2}(x, y)$,

we have $A_{i}(x, y)=\left[\left(I_{t}{ }^{,{ }^{2}}-I_{t}{ }^{\prime,}, \times I_{t}{ }^{\prime}\right) / \omega^{4}\right]^{1 / 2}$.

Because multiple phase-shifted images are taken in a time sequence, the derivatives have to be expressed by discrete differences of 4 sequential images $I_{l}(x, y) \sim I_{4}(x, y)$. Thus, the first, second and third difference of $I(x, y, t)$ can be given by

$I_{1}^{\prime}=I_{2}(x, y)-I_{l}(x, y)$;

$I_{1}{ }^{\prime \prime}=I_{3}(x, y)-2 I_{2}(x, y)-I_{l}(x, y)$;

$I_{l}{ }^{,,},=I_{4}(x, y)-3 I_{3}(x, y)+3 I_{2}(x, y)-I_{l}(x, y)$.

Therefore Eq. (27) becomes

$A_{i}(x, y)=\mathrm{C}\left\{\left[I_{3}(x, y)-2 I_{2}(x, y)-I_{l}(x, y)\right]^{2}-\left[I_{4}(x, y)-\right.\right.$

$\left.\left.3 I_{3}(x, y)+3 I_{2}(x, y)-I_{l}(x, y)\right]\left[I_{2}(x, y)-I_{I}(x, y)\right]\right\}^{1 / 2}$,

where $\mathrm{C}=1 / \omega^{4}$. Given that $\mathrm{C}$ is a constant and to avoid presence of complex value, a practical algorithm is proposed as

$A_{i}(x, y)=\mid\left[\left(I_{3}(x, y)-2 I_{2}\left(x, y-I_{1}(x, y)\right]^{2}-\left\{I_{4}(x, y)-3\left[I_{3}(x, y)-\right.\right.\right.\right.$

$\left.\left.I_{2}(x, y)\right]-I_{l}(x, y)\right\}\left.\left[I_{2}(x, y)-I_{l}(x, y)\right]\right|^{1 / 2}$.

Hence the tomography of $i^{\text {th }}$ layer of the sample can be solved without finding the phase-shift angle.

This algorithm is about 3 times faster than the traditional 4-step or 5-step algorithms. Compared to the traditional 4step based algorithm, this algorithm is more robust when the phase-shift angle is not stable. Because FF-OCT deals with images which have massive pixels, the speed of an algorithm 
becomes very important, when extracting the tomogram from OCT raw data.

\section{OPTICAL SYSTEMS FOR LARGE AREA FF-OCT}

There are couples of different configurations, which can be used for large area FF-OCT. Fig. (1a-d) show four types. Fig. (1a) is a Michelson free-space interferometer based system, where one lens is used as a collimator and another lens for imaging the sample to a camera. Fig. (1b) shows a system using a fibre imaging bundle [20], so that the sample arm becomes flexible. Fig. (1c) illustrates a system configuration where the sample is outside the interferometer and illuminated by both interferometer beams [2]. The scanning depth is determined by the difference of two distances $U$ and V. Fig. (1d) is a system configuration with a pair of CCD cameras, which make system capable of grabbing images with a phase-deference [12]. Two liquid crystal shutters are used to introduce $\pi / 2$ phase shift between images grabbed by two CCD cameras, as described by Eq. (13) in the previous section.

All these configurations are interferometer based systems, consisting of a broadband light source, reference arm and sample arm. To make a FF-OCT system, the computerized motion and vision modules are required, in which an area camera is used to grab the interference images and a translation stage with sub-micron accuracy is used for the depth scanning.

However, in practice, the lateral interference fringes may not be removed totally by using the equations mentioned above, because of the poor camera quality and the lack of repeatable accuracy of the motion motor. The CCD camera normally becomes saturated at the illuminated area and insensitive at the dark area. Due to these distortions, the para- sitic fringes will be introduced into the tomographic images retrieved. In addition, the series of images with different phase shift are grabbed at different times and the working environment may vary for each image. Among the factors affecting the images, the setting of the camera is critical. The shutter time and particularly the auto gain control affect the response attribute of the camera and can cause the generation of the parasitic fringes. Mechanically, the phase shift between sequential images may not be strictly equal, and a small angle error $\Delta \varphi$ may also be introduced. An effective method to reduce the parasitic fringes is the camera calibration. The calibration has to be done before doing the extraction of the tomogram.

\section{FF-OCT USING WHITE LIGHT SOURCE}

Currently, the mostly used broadband light source in OCT systems is the Superluminescent Diode (SLD). However, due to its relatively low power, it could not be used in applications where the sample has low reflection/scattering. Because of its high power and broad bandwidth, a white light source, e.g., tungsten halogen lamp or xenon short-arc lamp is a good alternative an SLD. A comparison of these lamps is given in [21]. Although a xenon lamp provides higher radiance than a halogen lamp, it can reduce contrast and produce artifacts in OCT images. In addition, its price is much higher than a halogen lamp. Given these factors, in our system, a halogen lamp was adopted. Fig. (2) shows the spectrum of a halogen white light source.

The white light source used in our FF-OCT system is Intralux 6000, halogen lamp. Its central wavelength is $650 \mathrm{~nm}$, bandwidth $200 \mathrm{~nm}$, average power 200 Watts. Its measured temporal coherence and spatial coherence properties are given in Fig. (3a,b), respectively. Given the bandwidth about $200 \mathrm{~nm}$ in Fig. (2), the depth resolution of the

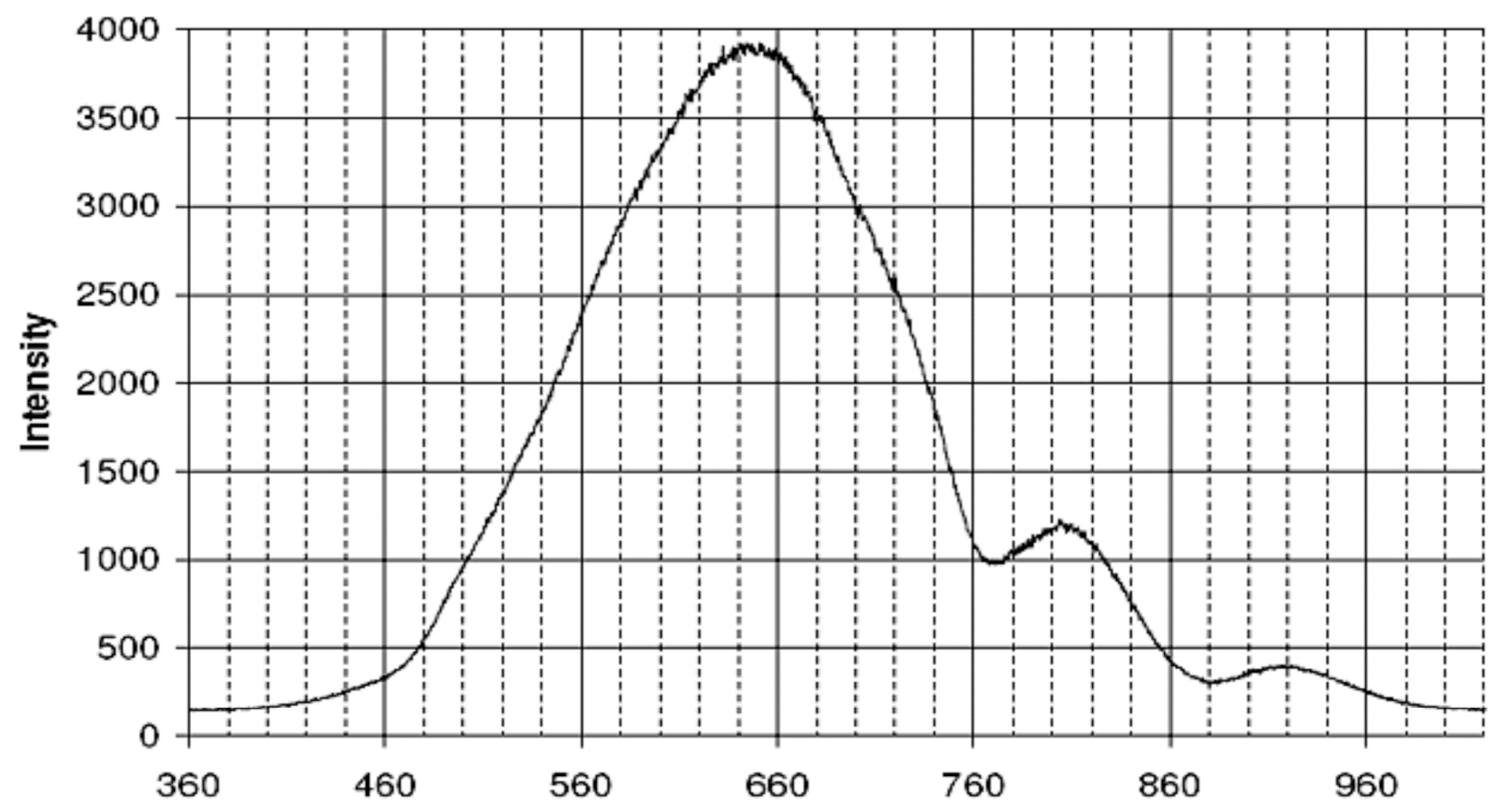

Fig. (2). Spectrum of a halogen white light source. 


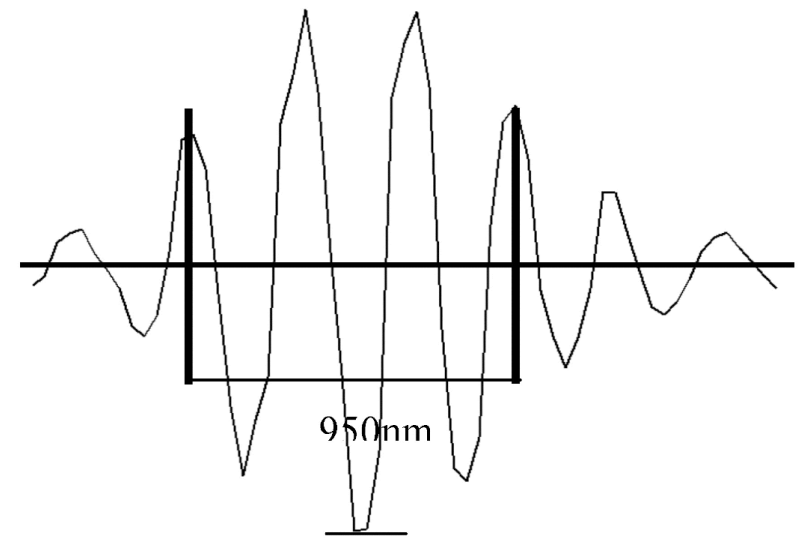

(a)

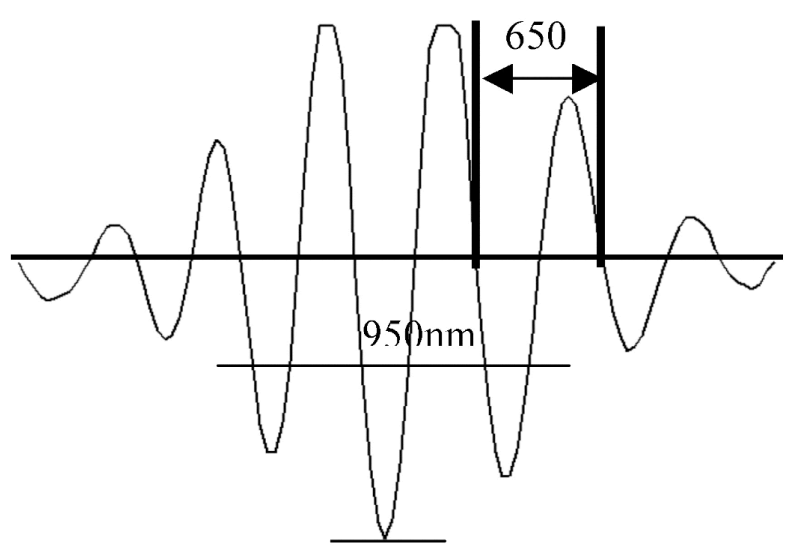

(b)

Fig. (3). Coherence characterization of halogen light source. (a) Temporal coherence measurement. (b) Spatial coherence measurement.

OCT system using this source is $930 \mathrm{~nm}$, according to Eq. (8).

The configuration of a large-field FF-OCT using white light source is shown in Fig. (1a) and the system is shown in Fig. (4). The specification of the system is given below:

Working area: $25 \mathrm{~mm}$ X $25 \mathrm{~mm}$;

Light source: Halogen lamp with central wavelength 700 $\mathrm{nm}$ and bandwidth $200 \mathrm{~nm}$;

Depth resolution: $0.9 \mu$ meter;

Frame rate: 30 frames / second;

Camera: $1024 \times 1024$ pixels at 12 bit gray levels (Dalsa 1M15);

Depth scanning accuracy: $37 \mathrm{~nm}$ (Thorlabs T25 translation stage).

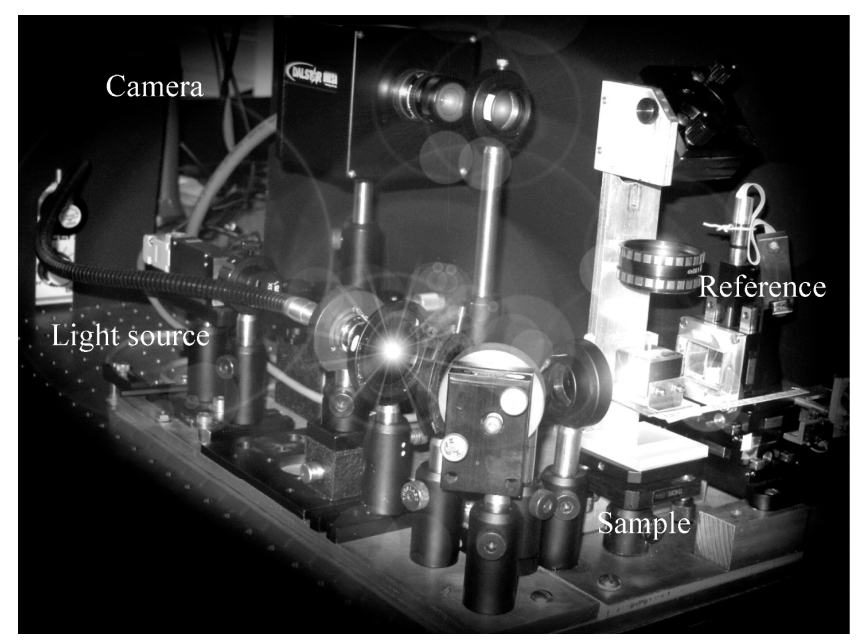

Fig. (4). FF-OCT system using halogen light source.

The alignment and tuning up is a critical procedure. A mirror is placed in the sample arm before performing this procedure. The orientations of the beam splitter, reference and sample mirrors must be adjusted precisely. A small aperture placed right after the collimator lens was temporarily used to help the adjustment. Thus, one can observe two spots grabbed by the camera. The adjustment is performed by repeatedly adjusting the beam-splitter and mirrors, until these two spots overlap together accurately. Because the coherence length is about $1 \mu \mathrm{m}$, searching the interference fringes of the white light FF-OCT becomes extremely difficult. To find these fringes manually is almost impossible. In our experiment, this procedure was performed by a computer controlled motion and vision system with an in situ image processing tool. The system scans and searches the possible interference fringes by analyzing the sequentially grabbed images. It stops the scanning at the position where the current image has the maximum contrast. Then the fine adjustments are performed at a slower and more accurate motion speed. A narrow interference-fringe pattern will appear at the central area of the grabbed image if the alignment is properly done, as shown in Fig. (5).

\section{APPLICATIONS}

\section{3D Intensity Imaging of an Object}

Although there are many interferometer based devices that could produce the profile of a 3D object, they lose the intensity information of the object, because the profilograph is obtained from the phase variable. The OCT based technology could generate an image at different heights of the object. In this case, even if the light can not penetrate through the object, such as metal surface, the image produced is the 3D intensity image of the object. Fig. (6) shows a set of height-layer segmented images, which represent the intensity images at different layers of a Canadian dollar coin. The separation between layers is $20 \mu \mathrm{m}$ in our experiments. Fig. $(\mathbf{6 j})$ is the direct background image of the coin, Fig. (6a-h) are 8 images extracted at 8 layers. Fig. (6i) is the summation of all 8 segmented images, it shows an entire side-view portrait, differing from that in Fig. $(\mathbf{6 j})$.

\section{Multiple-Layer Extraction Using White Light FF-OCT}

Because OCT has a resolution in the order of microns and the ability of peeling cross-sectional images from the 


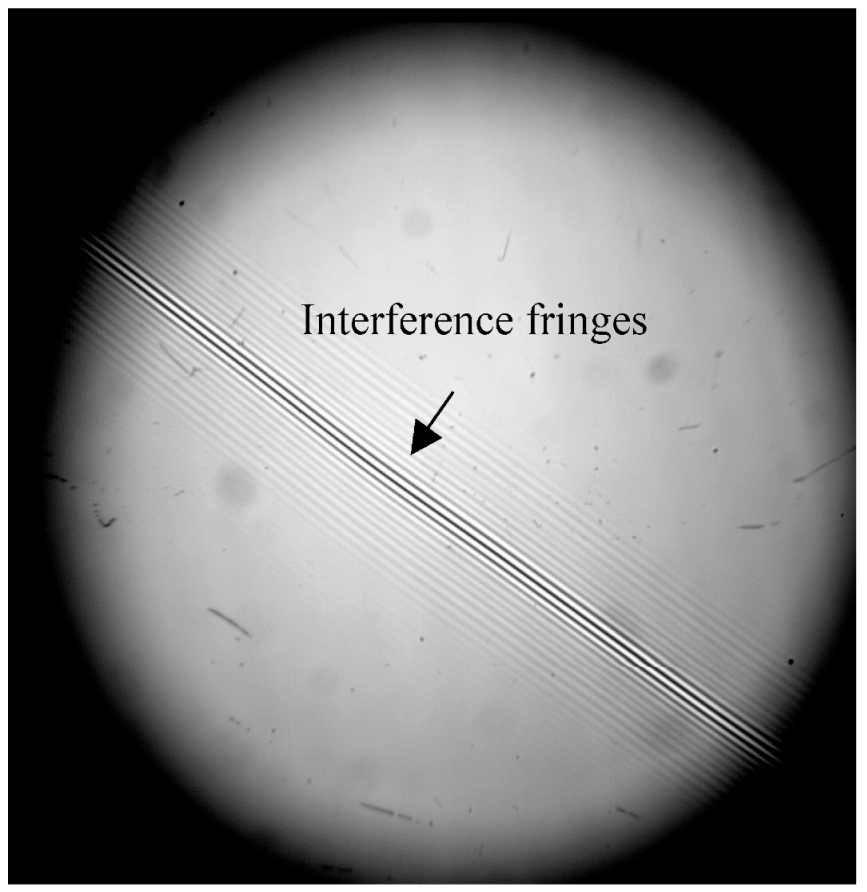

Fig. (5). FF-OCT white light interference fringes.
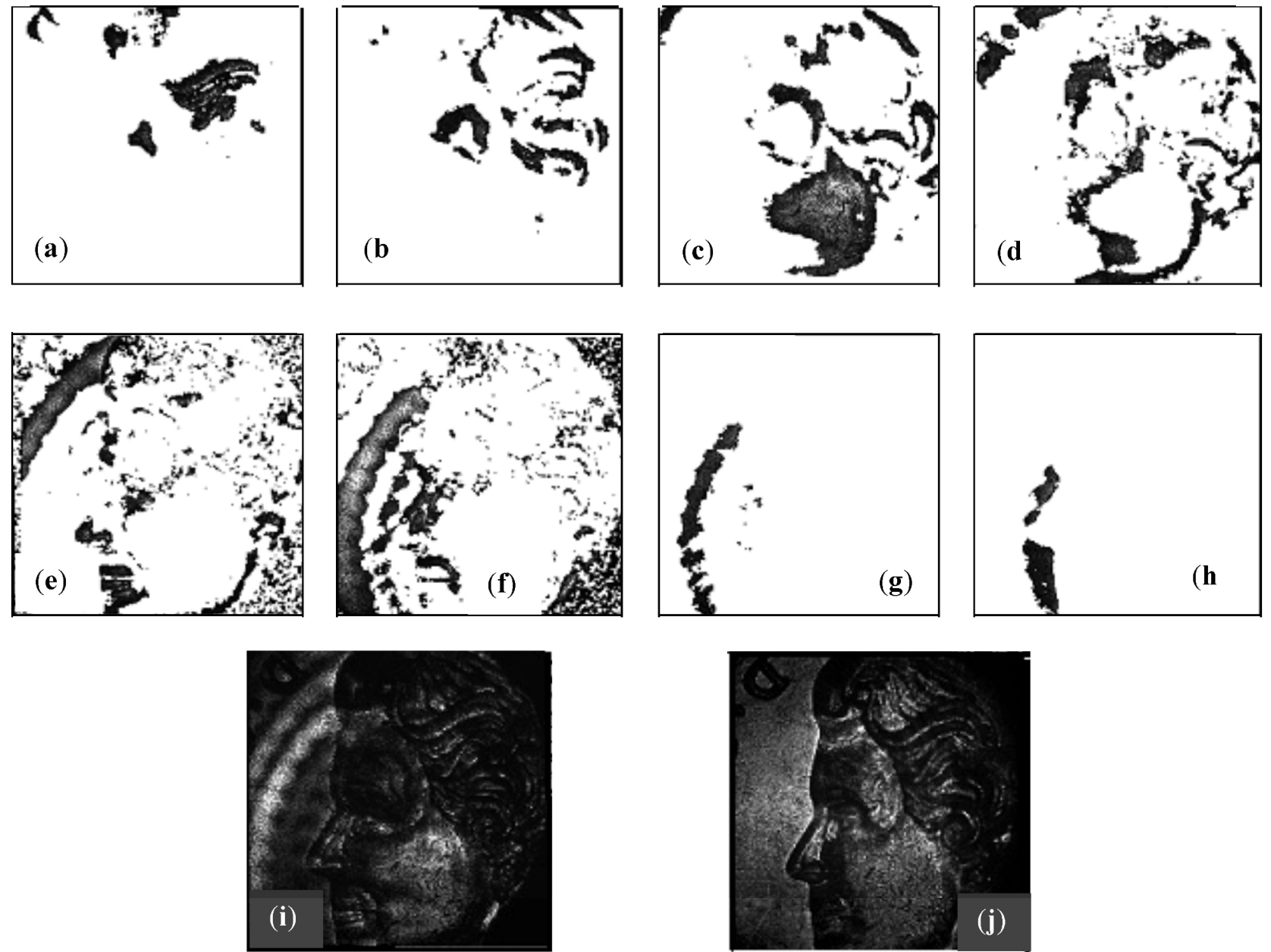

Fig. (6). 3D intensity images of a Canadian Looney segmented by FF-OCT. (a-h) images segmented at different heights, layer separation: 20 $\mu \mathrm{m}$. (i) Summation of images (a-h). (j) Direct imaging of Canadian Looney. 


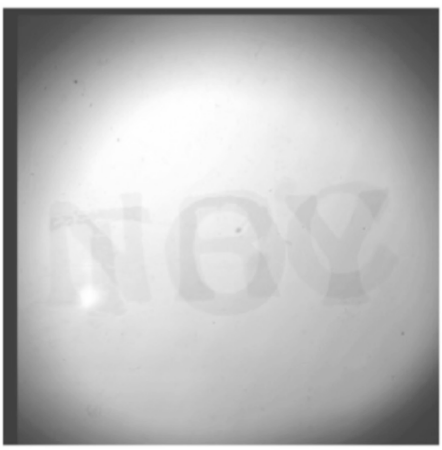

(a)

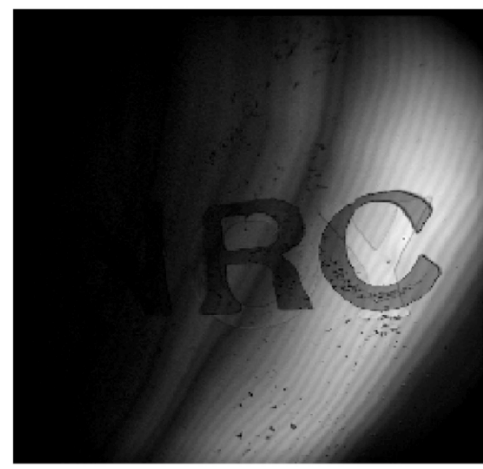

(d)

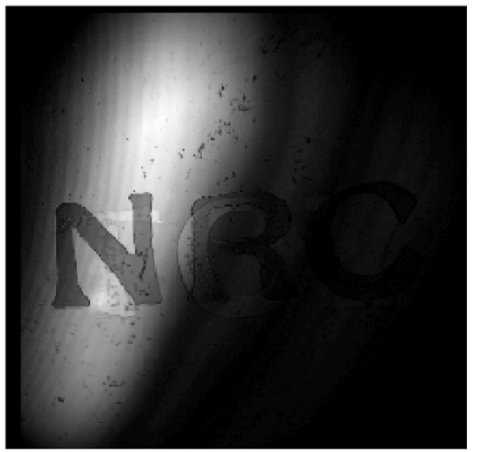

(b)

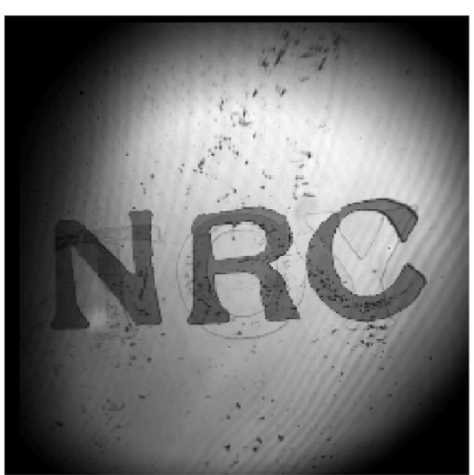

(e)

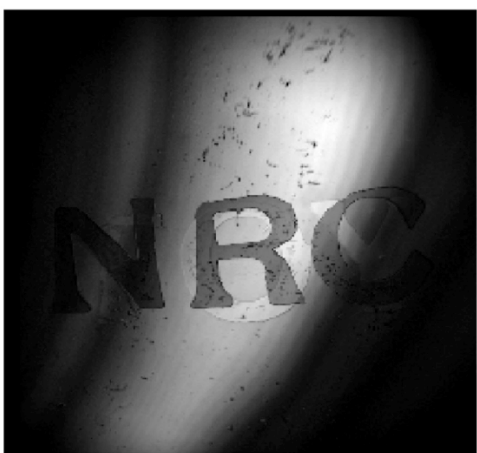

(c)

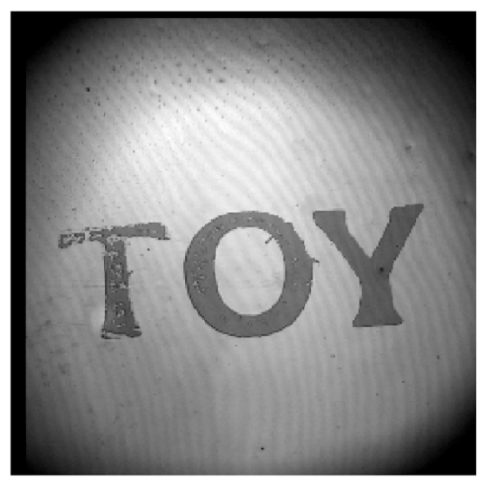

(f)

Fig. (7). Two layer information extraction by white light FF-OCT. (a) Direct imaging of the sample. (b-d) tomography images extracted at different depths. (e) The first and; (f) second layer images extracted.

when an artificial finger gummy that has an identical 2D pattern to a real fingerprint is taken as the input sample, those technologies can not detect the difference. The artificial fingerprint gummies create serious problems for the existing fingerprint recognition systems, as they can be cheaply made and easily applied to deceive the fingerprint system [22].

As the artificial fingerprints are normally made by translucent materials, FF-OCT could be a powerful tool to fast and effective recognition of such dummies. FF-OCT can

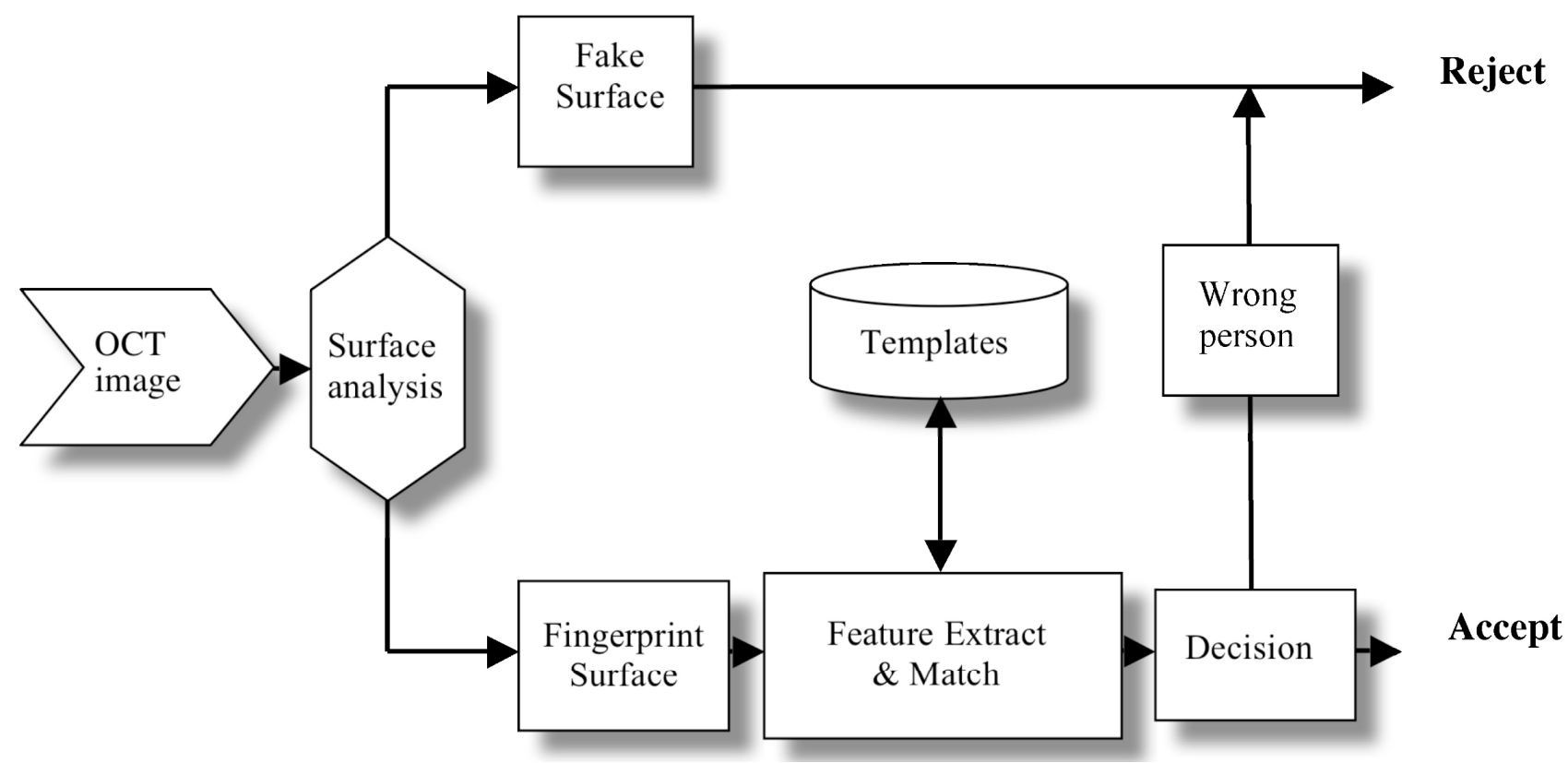

Fig. (8). Flow-chart of an anti-spoof fingerprint recognition system. 


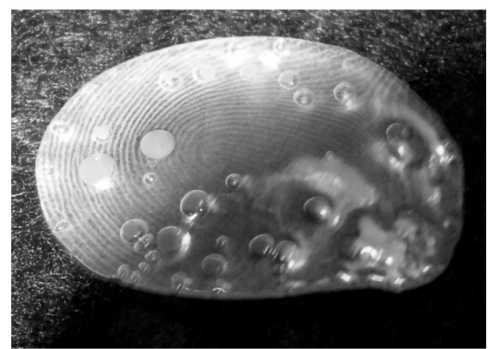

(a)

Fig. (9). (a) An artificial fingerprint dummy and; (b) its 2D direct imaging.
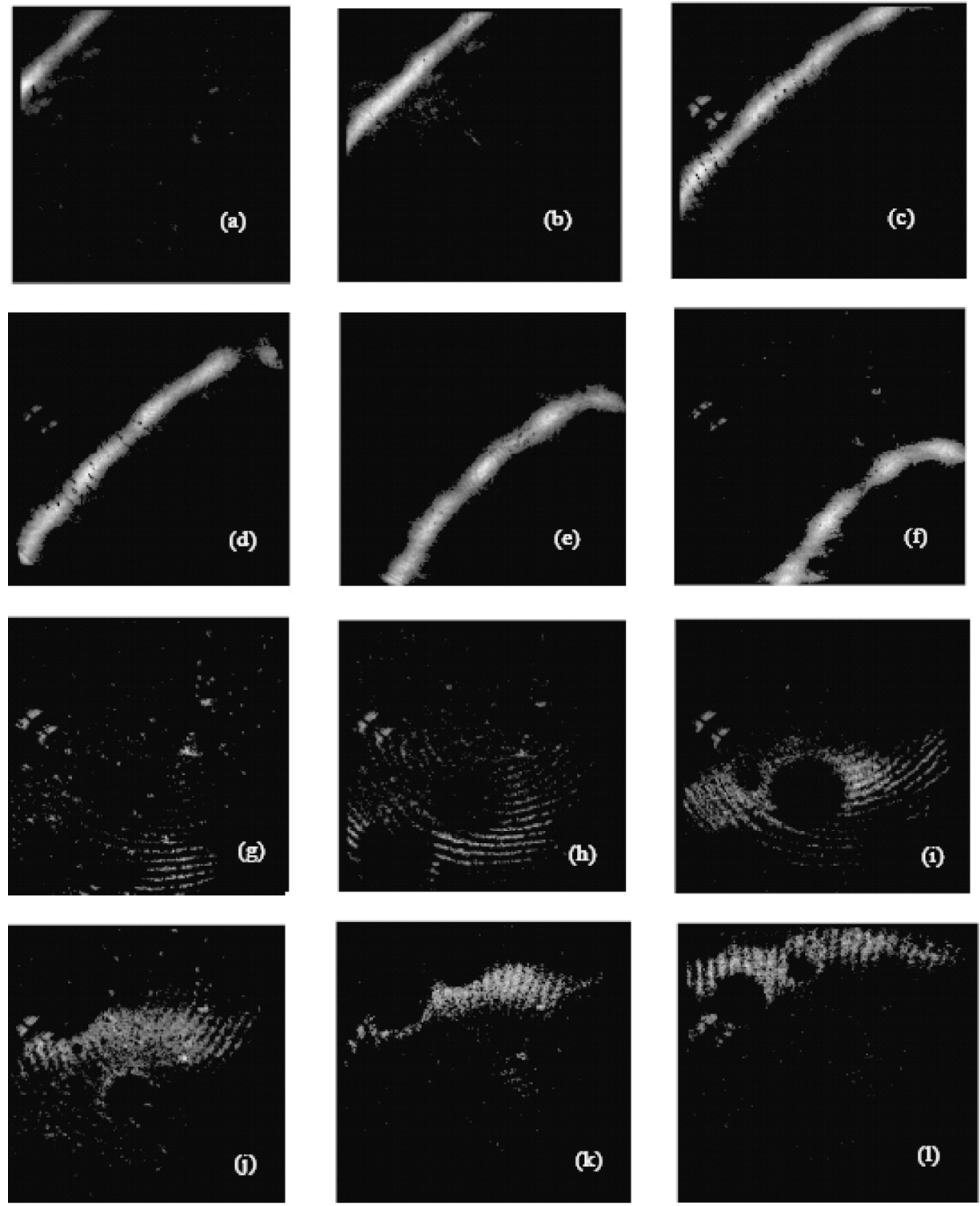

Fig. (10). Tomographic images of a dummy fingerprint. (a-f) images from the first surface (smooth surface). (g-l) images from the second surface (fingerprint surface).

detect both surfaces of a dummy: fingerprint surface and non-print surface, which do not exist in a real finger. In addition, an FF-OCT can explore the internal structure between these two surfaces, which is also different from a real finger. Fig. (8) show an anti-spoof fingerprint recognition system.
The dummy shown in Fig. (9) has successfully deceived a fingerprint scanning system (Microsoft Fingerprint Reader). A FF-OCT system was used to grab 3D tomography images of this gummy. Fig. (10) shows 2 groups of cross-sectional images obtained from experiments. During the depth scanning, two obvious surfaces of the dummy were 


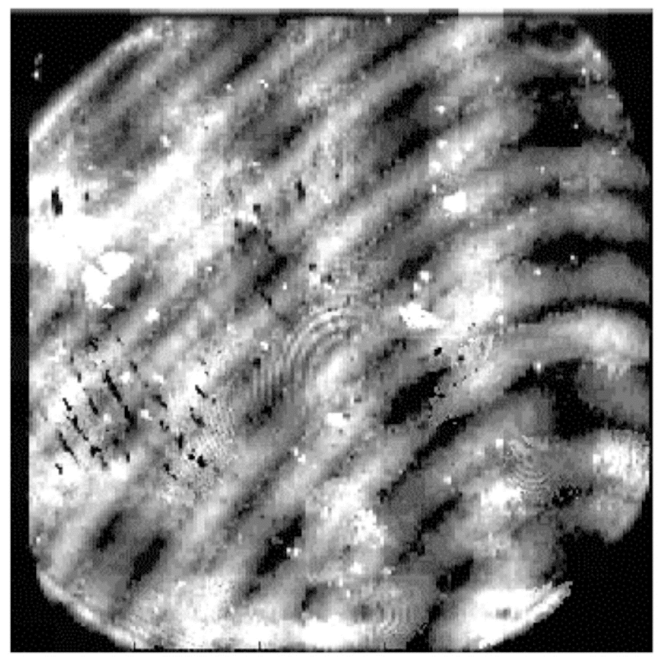

(a)

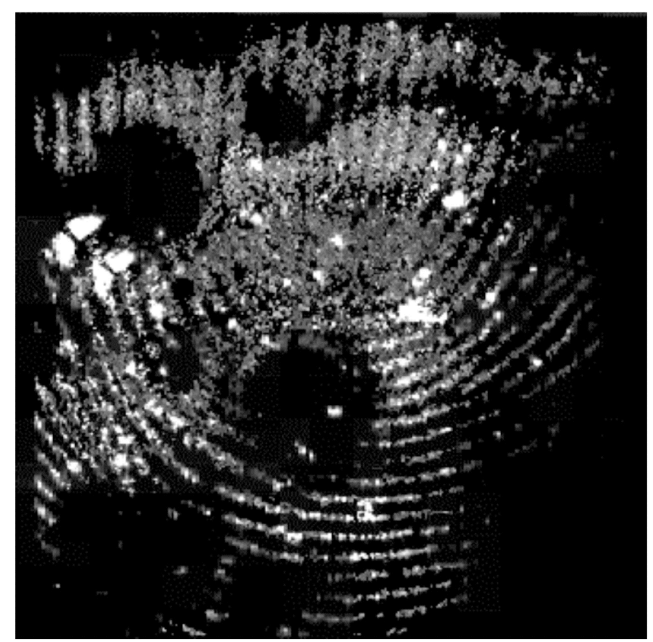

(b)

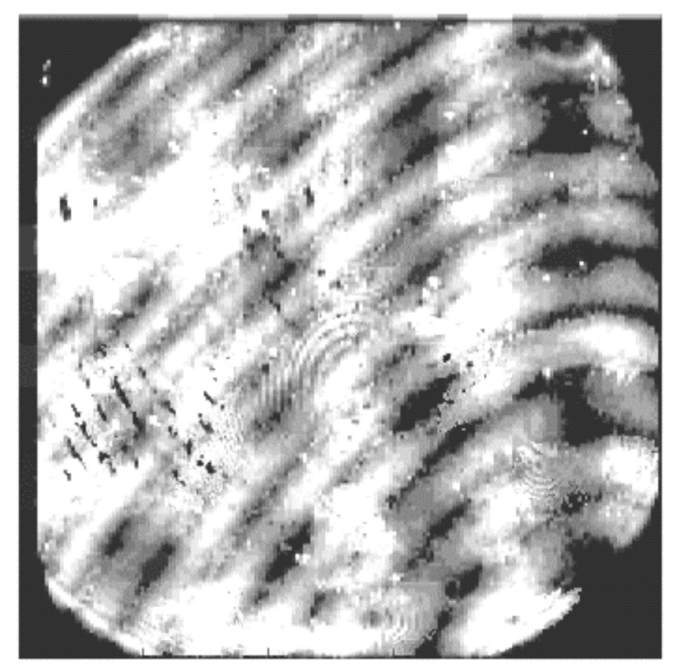

(c)

Fig. (11). Summations of tomographic images. (a) Summation of those in the first surface; (b) Summation of those in the second surface. (c) Summation of those in both surfaces.

found that exhibited different features. The outer surface shows a smooth 2D curve, as shown in Fig. (10a-f), which does not exist in the real finger; however, the inner surface shows segmented fingerprints at different layers, by Fig. (10g-l). The presence of and the patterns of those two surfaces prove that the sample under test is an artificial fingerprint dummy. Actually, between these two surfaces, there are many other layers that provide internal structures of the fake fingerprint. As the information on the two surfaces is enough to verify the fake fingerprint, all images of the internal layer are omitted in this paper.

The sum of the images in Fig. (10a-f) is given by Fig. (11a), which is a bright area without any fingerprints. (As the image sampling separation is somewhat broad, $40 \mu \mathrm{m}$, fringes appear when they are overlapped). Fig. (11b) shows the sum of these segmented fingerprints, as shown in Fig. (10g-I). Fig. (11c) demonstrates the sum of all those tomograms, which completely destroys the fingerprint, and is totally different from the image captured by a 2D camera commonly used in a fingerprint recognition system (Fig. 9b).

Fig. (12) provides another set of rotated 3D volume data. The red parts show the internal structure of the dummy, which are totally different to the internal tissues of a real finger. The presence of them and the patterns of both two surfaces prove that the object is an artificial fingerprint dummy. 


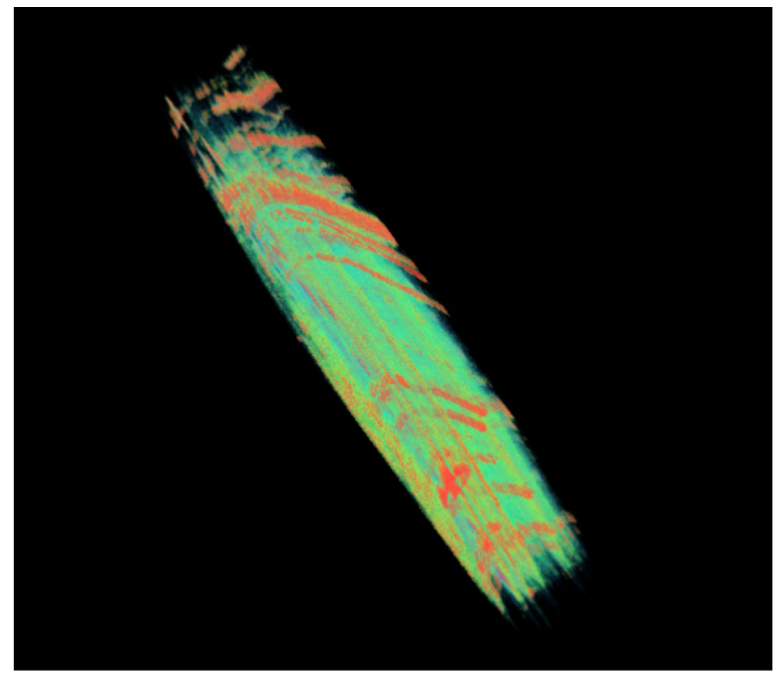

\section{DISCUSSION AND CONCLUSIONS}

In this paper, we have described a large-area FF-OCT and both its system and algorithm design. Unlike OCT systems working with point-scanning devices, the main consideration for FF-OCT system is the signal/noise ratio since the input power is spread to a large area. However, in applications where the samples are near transparent or translucent, FF-OCT could be a useful tool. White light FF-OCT can provide a powerful light source for the large area and deep exploration, but its wavelengths are basically in the visible band. For some applications, particularly biological samples, the useful wavelength windows are in infrared range. In addition, because the white light source has a much broader spectrum than SLDs, dispersion becomes a more serious issue than that arising from the system using SLD. A compensation procedure must be given if the sample has relatively large volume, by either hardware or digital techniques.

\section{REFERENCE}

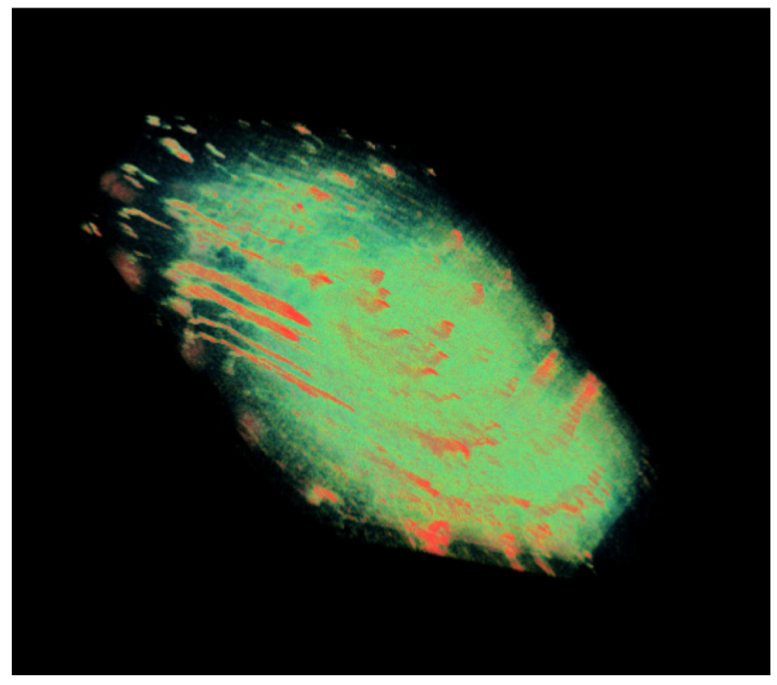

Huang E. A, Swanson C. P, Lin J. S, Schman W. G, Stinson W, Chang M. R, Hee T, Flotte K, Gregory C, Pullafito A, Fujimoto J. G. Optical Coherence Tomography. Science 1991; 254: 1178-81.

[2] Fercher1 A F, Drexler W, Hitzenberger C K, and Lasser T. Optical coherence tomography-principles and applications. Rep Prog Phys 2003; 66: 239-303.

[3] Fercher A. F, and Hitzenberger C. K. Optical coherence tomography. In: Wolf E. Progress in Optics, Elsevier Science B.V. 2002.

[4] Bouma B. E, and Tearney G. J. Handbook of Optical Coherence Tomography. Marcel Dekker: New York; 2002.

[5] Liu B, Brezinski M E. Theoretical and practical considerations on detection performance of time domain, Fourier domain, and swept source optical coherence tomography. J Biomed Opt 2007; 12: 044007-1 -12.

[6] Fercher A. F, and Hitzenberger C. K, Kamp G, and El-Zaiat S. Y. Measurement of intraocular distances by backscattering spectral interferometry. Opt Commun 1995; 117: 43-8.

[7] Hausler G. and Lindner M W. "Coherence radar" and "spectral radar" - New tools for dermatological diagnosis. J Biomed Opt 1998; 3: 21-31

[8] Choma M. A, Sarunic M. V, Yang C. H, and Izatt J. A. Sensitivity advantage of swept source and Fourier domain optical coherence tomography. Opt Express 2003; 11: 2183-9.

[9] Chinn S. R, Swanson E. A, and Fujimoto J. G. Optical coherence tomography using a frequency-tunable optical source. Opt Lett 1997; 22: 340-42.

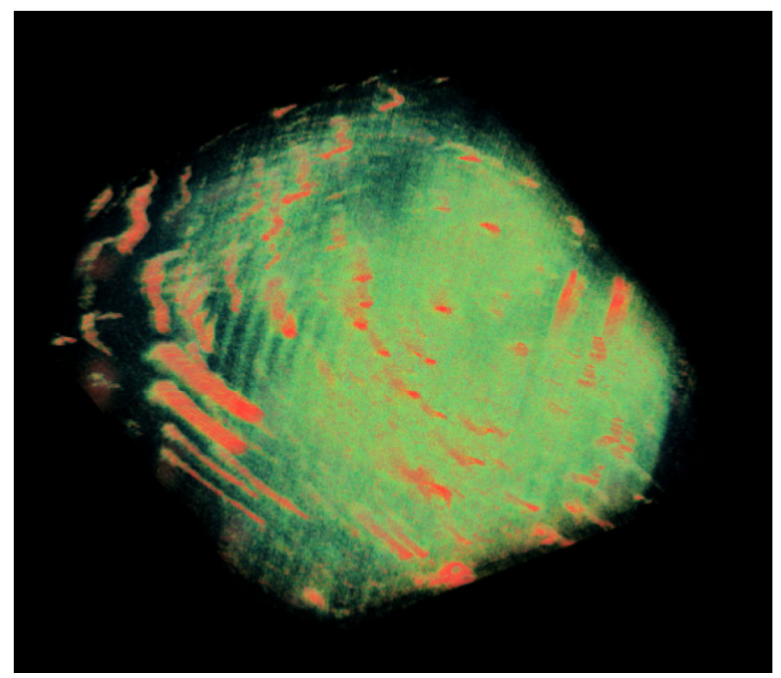

[10] Beaurepaire E A, Boccara C., Lebec M, Blanchot L, and SaintJalmes H. Full-field optical coherence microscopy. Opt Lett 1998; 23: 244-6.

[11] Bourquin S, Seitz P, and Salathe R. P. Optical coherence topography based on a two-dimensional smart detector array. Opt Lett 2001; 26: 512-514.

[12] Akiba M, Chan K. P, and Tanno N. Full-field optical coherence tomography by two-dimensional heterodyne detection with a pair of CCD cameras. Opt Lett 2003; 28: 816-818.

[13] Chang S, Liu X, Cai X, and Grover C. P. Full-field optical coherence tomography and its application to multiple-layer 2D Information retrieving. Opt Commu 2005; 246: 579-585.

[14] Greivenkamp J. E, and Bruning J. H. Phase Shift Interferometer In: Optical Shop Testing ( $2^{\text {nd }}$ Edition $)$, John Wiley \& Sons. New York. 1992

[15] Carré P. Installation et Utilization du Comparateaur Photoelectrique et Interferentiel du Bureau International des Poids de Mesures. Metrologia 1966; 2: 13.

[16] Hariharan P, Orebm B. F, and Eiju T. Digital Phase-Shifting Interferometry: A simple Error-Compensating Phase Calculation Algorithm. Appl Opt 1987; 26: 2504.

[17] Dubois A, Vabre L, Boccara AC, Beaurepaire E. High-resolution full-field optical coherence tomography with a Linnik microscope. App Opt 2002; 41: 805-12.

Fig. (12). Three 3D images from the rotating 3D dummy fingerprint. 
[18] Dubois A. Phase-map measurements by interferometry with sinusoidal phase modulation and four integrating buckets. JOSA A, 2001; 18: 1972-9.

[19] Chang S, Cai X, Flueraru C. An efficient algorithm used for fullfield optical coherence tomography. Opt Laser Eng 2007; 45: 11706.

[20] Ford H. D, and Tatam R. P. Full-field coherence tomography. Proc SPIE Vol. 58580J-1. 2005.
[21] Fercher A. F, Hittzenberger C, Sticker M, Moreno-Barriuso E, Leitgeb R, Drexler W, and Sattmann H. A thermal light source technique for optical coherence tomography. Opt Comm 2000; 185: 57-64.

[22] Matsumoto T, Matsumoto H, Yamada K, and Hoshino S. Impact of artificial "gummy" fingers on fingerprint systems. Optical Security and Counterfeit Deterrence Techniques IV. Proc. SPIE 4677, 275289. Rudolf L. van Renesse, Editor, Proceedings of SPIE Vol. 4677 (2002) (C) 2002. 